\title{
Variation of dependence of the cusp location at different altitude on the dipole tilt
}

\author{
GUO JianGuang ${ }^{1,2}$, SHI JianKui ${ }^{1 *}$, CHENG ZhengWei ${ }^{1}$, ZHANG ZiYing ${ }^{1}$, WANG Zheng ${ }^{1}$, \\ ZHANG TieLong ${ }^{3}$, LIU ZhenXing $^{1} \&$ DUNLOP Malcolm ${ }^{4}$ \\ ${ }^{1}$ State Key Laboratory of Space Weather, Center for Space Science and Applied Research, Chinese Academy of Sciences, Beijing 100190, China; \\ ${ }^{2}$ National Satellite Meteorology Center, Beijing 100081, China; \\ ${ }^{3}$ Space Research Institute, Austrian Academy of Sciences, Graz, A-8042, Austria; \\ ${ }^{4}$ Rutherford Appleton Laboratory, Chilton, Didcot, OX11 OQX, UK
}

Received December 19, 2012; accepted February 25, 2013; published online May 3, 2013

Using the Cluster cusp crossings data, dependence of the cusp location at the mid-altitude on the geomagnetic dipole tilt during northward IMF is studied. The results show that the cusp center moves $0.051^{\circ}$ Invariant Latitude (ILAT) upon the increase of $1^{\circ}$ in the dipole tilt angle at the average altitude of $5.8 \mathrm{R}_{\mathrm{E}}$ (Earth radius). According to the present results obtained at the altitude of the Cluster orbit and previous results obtained at other altitudes of other satellite orbits, it is found that the higher the altitude in the cusp region is, the bigger the dependence of cusp location on the dipole tilt angle will be. If the altitude increases by $1 R_{E}$ in the cusp region, the dependence will increase by $0.012^{\circ}$ ILAT upon the increase of $1^{\circ}$ in the dipole tilt angle. Some possible physical mechanisms are discussed and it shows that the cusp location will be more sensitive to the solar wind dynamic pressure if the altitude is high.

cusp location, dependence, dipole tilt, altitude variation, different satellite

Citation: $\quad$ Guo J G, Shi J K, Cheng Z W, et al. Variation of dependence of the cusp location at different altitude on the dipole tilt. Chin Sci Bull, 2013, 58: 3541-3545, doi: 10.1007/s11434-013-5831-1

As the solar wind interacts with the magnetosphere, the two cusps always change in their locations and shapes. Through the two narrow funnel-shaped cusp regions, the magnetosheath plasma can directly access to the high-latitude ionosphere $[1,2]$. So, these two cusp regions are generally recognized as the key regions for the solar wind-magnetosphere-ionosphere coupling [3]. As the geomagnetic dipole axis has an angle to its spin axis, the ram pressure of the solar wind, the IMF orientation (i.e. magnetic field component $B_{y}$ and $B_{x}$ ), and the $\mathrm{S}_{\mathrm{q}}$ current system around each cusp region in the magnetopause are likely to have some effects on the position of the cusp $[4,5]$.

It has been theoretically predicted that the cusp would be several degrees lower in the hemisphere whose geomagnetic dipole axis points away from the solar wind ram velocity

*Corresponding author (email: jkshi@nssc.ac.cn) direction [5]. Based on the OGO4 data (700 km of altitude), Burch [6] first investigated the effect of the dipole tilt angle on the cusp equatorward boundary, and found a cusp shifted by approximately $4^{\circ}$ in the invariant latitude (ILAT) over the total yearly range of the dipole tilt angles. A survey of the cusp crossings observed by DMSP F7 ( $\sim 800 \mathrm{~km}$ of altitude) has shown that in response to the changing dipole tilt angle, the cusp is found to move by $\pm 2^{\circ}$ magnetic latitude (MLAT) from the mean position, which is located between $75.9^{\circ}$ and $76.8^{\circ}$ MLAT [7].

The cusp has also been studied with the data from high-altitude and mid-altitude satellites. Němeček et al. [8] found the footprint positions of the cusp-like plasma, which is determined by the observation of the MAGION-4 satellite (altitudes $5-15 \mathrm{R}_{\mathrm{E}}$ ), have a substantial latitudinal dependence on the dipole tilt angle with a slope of $0.15^{\circ}$ MLAT per $1^{\circ}$ of the dipole tilt angle. This slope increases to $0.16^{\circ}$ 
MLAT per $1^{\circ}$ in the vicinity of the magnetopause. The observations of the Polar Spacecraft (at altitudes 5-10 $\mathrm{R}_{\mathrm{E}}$ ) suggested the same dipole tilt angle effect on the location of the cusp with roughly $0.07^{\circ}$ ILAT for every $1^{\circ}$ of the dipole tilt angle at mid-altitude [9]. In a recent paper, Pitout et al. [10] conducted a statistical study of the mid-altitude cusp using the four years Cluster crossings of at the average altitude of about 5.6 $\mathrm{R}_{\mathrm{E}}$. Their results showed that an increase of about $11^{\circ}$ in the dipole tilt angle produces an increase of $1^{\circ}$ in ILAT for the poleward boundary of the cusp, rather than the centre of the cusp region. Newell et al. [11] checked the difference between the two hemispheres in the dipole tilt angle effect by the DMSP satellite observations at the $\sim 800 \mathrm{~km}$ altitude. They gave a slope of $0.043^{\circ}$ cusp latitude per $1^{\circ}$ dipole tilt angle by a large number of cases (1857), mostly from the Southern Hemisphere. The smaller subset of data from Northern Hemisphere (407) shows a slope of $0.046^{\circ}$ cusp latitude per $1^{\circ}$ dipole tilt angle, and they concluded that Northern and Southern Hemispheres act similarly.

In this paper, identifying the cusp center with the Cluster data, we study the cusp location dependence on the dipole tilt angle. Then, considering both our new result and previous results of the dependences of cusp location at different altitudes on the dipole tilt together, we find that the dependence of cusp location on the dipole tilt increases with the altitude of the cusp region monotonously.

\section{Method and data presentation}

One way to discern the cusp region is by a diamagnetic depression [12]. We can expect to see a decrease of the total magnetic field and some fluctuations in the magnetic field because of the diamagnetic effects of the incoming magnetosheath plasma and the weak background magnetic field in the mid-altitude cusp region [13-15]. We can also expect to see the magnetosheath-like (high density and low energy) plasma and an increasing electron density in this region $[9,16]$. Based on these expectations and the satellite data that we have, the criteria to identify the cusp region are as follows: a decrease of the magnetic field strength from the background field greater than $1 \mathrm{nT}$ associated with some fluctuations; a sudden increase in the proton and electron density (greater than $5 \mathrm{~cm}^{-3}$ ); an electron thermal energy less than $100 \mathrm{eV}$; and the presence of significant $\mathrm{He}^{++}$ (greater than $0.5 \mathrm{~cm}^{-3}$ ) which signifies a solar wind origin. Some authors have used these conditions to identify the cusp region with the Cluster data and Polar satellite data $[9,17]$. In our study, we use these conditions to identify the two cusp edges firstly. Then, we take the cusp center as the middle of the two edges. The Cluster magnetic field, ion and electron data are from the Flux Gate Magnetometer (FGM) [18], Cluster Ion Spectrometer (CIS) [19], and Plasma Electron and Current Experiment (PEACE), respec- tively [20].

It is well known that the interplanetary magnetic field (IMF) plays a significant role in controlling the cusp location. For increasingly southward IMF, the cusp location is observed to move to lower latitudes, while for increasingly northward IMF, the cusp displays a very slight tendency to move to higher latitudes. Therefore, to limit the contribution of the IMF, we investigated the cusp location dependence on the dipole tilt angle only for northward IMF cases, for which conditions the cusp is almost immune to the IMF variation $[7,10,21]$. In this study, the ACE IMF $B_{Z}$ data with time resolution of 1 minute are used and from the NASA's Space Physics Data Facility (SPDF).

\section{Results and discussion}

With criteria and instruments mentioned above, we obtain a data set containing 95 Cluster cusp crossings at the average altitude of $5.8 \mathrm{R}_{\mathrm{E}}$ during the northward IMF.

\subsection{Dependence of cusp location on the dipole tilt at Cluster orbit}

The dipole tilt angle is defined as the angle between the north dipole axis and the GSM Z-axis (Figure 1). This angle is positive when the dipole tilts toward the Sun, and negative when the dipole tilts away from the Sun. Here we just multiply the dipole tilt angle by -1 for the southern cusps.

Cluster, with a polar orbit, crosses both the northern and southern cusp regions each year. Since apogee lies slightly south of the equator, the southern crossings are sampled at slightly higher altitudes than the northern crossings and the local time coverage is also slightly different. We note that there are two most closely related studies to the present work, which adopted slightly different criteria. Zhou et al. [9] used the ILAT values, by mapping from the T89 model,

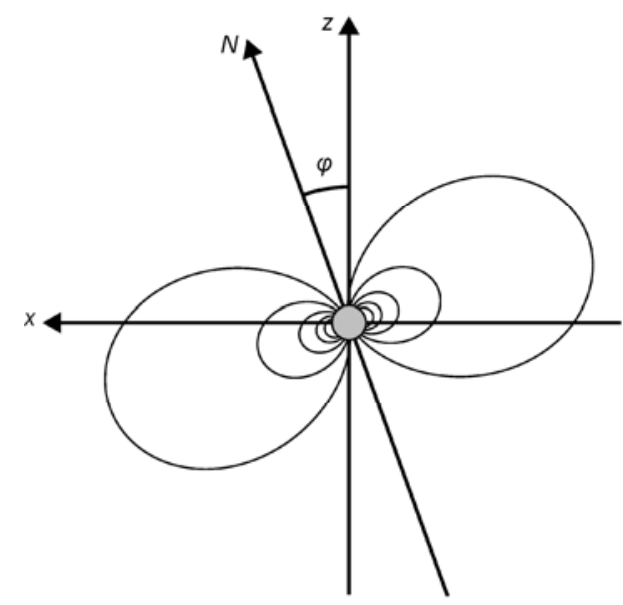

Figure 1 The dipole tilt angle $\varphi$ is defined as the angle between the north dipole axis marked with $N$ and the GSM $z$-axis. 
to centre the cusp crossings to the implied magnetic funnel from the field model. Pitout et al. [10] did not use the mid cusp region to identify position, but used the poleward boundary in order to better reveal the effects of northward and southward IMF $B_{z}$. In the first case, the use of T89 shifts the effective ILAT values of each position higher, but we expect the dipole tilt dependence to be similar. In the second case, it is possible that the poleward boundary has a higher dependence on the dipole tilt. However, all of the previous author, except Pitout et al. [10] identified the center of the cusp location to study the dependence of cusp location on geomagnetic dipole tilt $[8,9,11]$. Also, the previous authors studied the dependences for the northern and southern cusp crossings together. In order to compare our result with the previous results, we also identify the center of the cusp location to study the dependence of cusp location on the dipole tilt, and consider both the northern and southern cusp crossings together.

Figure 2 shows the centers of the cusp crossings of the Cluster from 2001 to 2005 with their ILAT and the corresponding dipole tilt angle. From Figure 2, we can see that the northern and southern crossings are both considered together, and there are 95 cusp crossings during northward IMF. From Figure 2 we can see that ILAT of the cusp center is increasing with the tilt angle.

The fit line of the center of the cusp crossings versus the dipole tilt in Figure 2 is linear and conforms to the following relation:

$$
\Lambda=77.93+0.051 \varphi \text {. }
$$

Here $\Lambda$ is the ILAT of the cusp center and $\varphi$ is the geomagnetic dipole tilt angle.

From eq. (1), we can see that the mean cusp position (for $0^{\circ}$ tilt angle) is $77.93^{\circ}$ ILAT. The slope of the fit line is $0.051^{\circ}$. This means that the cusp moves $0.051^{\circ} \mathrm{ILAT}$ for every $1^{\circ}$ increase in the dipole tilt angle at an average altitude of $5.8 \mathrm{R}_{\mathrm{E}}$ (corresponding to the altitude from 4 to $8 \mathrm{R}_{\mathrm{E}}$ ). The overall behavior of the cusp is the same as that reported by some previous authors $[8,9,11]$, i.e. the slope is positive, which implies that the greater the dipole tilt angle is, the higher the ILAT of the cusp center will be.

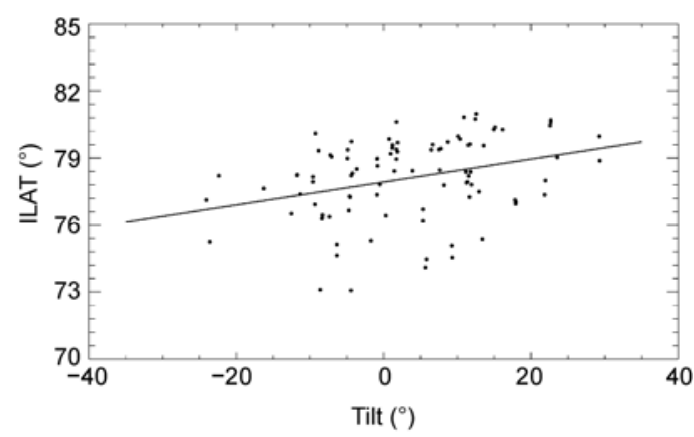

Figure 2 The ILAT of the cusp crossing of Cluster from 2001 to 2005 versus geomagnetic dipole tilt angle during the northward IMF.
Pitout et al. [10] used the Cluster data to identify the poleward boundary of the cusp at the average altitude of about 5.6 $\mathrm{R}_{\mathrm{E}}$ and studied the dependence of the cusp location on the dipole tilt. The way used in their study is similar to that for Figure 2. They obtained a slope of $0.091^{\circ}$ for the fit line. In our study, we focus on the center of the cusp, and the average altitude of the cusp crossings is $5.8 \mathrm{R}_{\mathrm{E}}$ and the slope is $0.051^{\circ}$. Our result has a difference of $0.040^{\circ}$ in slopes from the result obtained by Pitout et al., which implies the cusp boundary is more influenced than the cusp center.

\subsection{Variation of the dependency}

As we mentioned above, some authors have studied the dependence of cusp locations on the dipole tilt from other different satellite observations at different altitudes. If we consider both the previous dependences obtained at different altitudes and our results in this paper together, we can find that the higher the altitude of the cusp crossing is, the larger the slope will be [8-11].

Figure 3 summarizes the dipole angle tilt dependence of the cusp location in terms of the altitude. The dependences (for example, the slop in eq. (1)) observed in this study and previous studies are represented by open circles. Point 1 is from Newell et al. [11], point 2 from Pitout et al. [10] (we should note that point 2 in Figure 3 is placed high, compared to the other studies, since they used the poleward boundary of the cusp, rather than the centre of the cusp region), point 3 from this paper, point 4 from Zhou et al. [9] and points 5 and 6 from Němeček et al. [8]. The altitudes of the points in Figure 3 are just the typical values. From Figure 3 , if we only consider the cusp center (i.e. except point 2 ), we can get a conclusion that the dependence increases monotonically with the increasing altitude.

In Figure 3, when we fit the points, except the point 2, that obtained by different authors from satellites at different altitudes of the cusp region, a linear relation can be written as

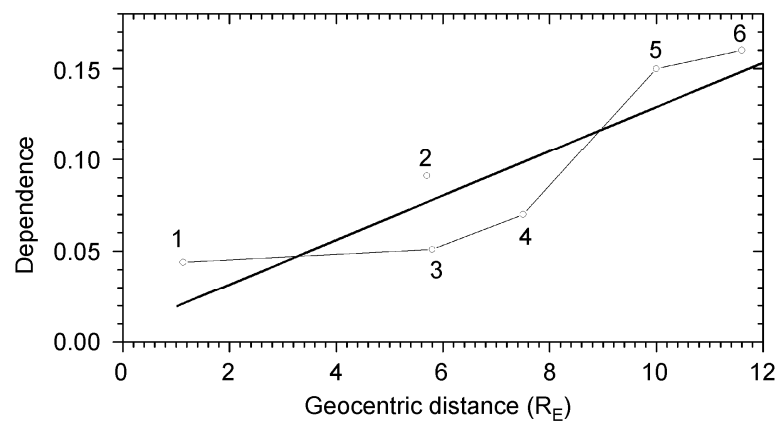

Figure 3 The dependence of cusp location on the dipole tilt angle at different altitudes. Points 1, 2, and 3 are from Newell et al. [11], Pitout et al. [10] and this paper, respectively, and point 4 from Zhou et al. [9]. Points 5 and 6 are from Nĕmecĕk et al. [8]. The linear line is the fitted line for points $1-6$ except point 2 . 


$$
k=0.012 r+0.0074 .
$$

Here, $k$ is the dependence of cusp location on the dipole tilt angle in unit of degree per degree and $r$ is the geocentric distance of the cusp region with a unit of $\mathrm{R}_{\mathrm{E}}$. Eq. (2) means that the dependence of cusp location on the dipole tilt angle will increase 0.012 degree per degree when the altitude of the cusp region increases by $1 R_{E}$.

Here point 2 is excepted because the dependence obtained by Pitout et al. [10] is for the poleward boundary of the cusp, rather than the centre of the cusp region. Indeed, the fit line in Figure 3 is quite raw. The real fit curve needs to be studied further with more observations. However, it should be reliable that the dependence increases monotonically with the increasing altitude.

\subsection{Discussion}

The solar wind interaction with magnetosphere has a significant influence on the cusp location besides the magnetosphere-ionosphere coupling [3,22]. The ram pressure of the solar wind or the IMF orientation (i.e. $B_{y}$ and $B_{x}$ ) could take effect on the cusp location directly. In this case, the cusp boundaries (both poleward and equatorward) are more sensitive to the ram pressure of the solar wind or the IMF orientation. Furthermore, the cusp tends to widen during negative tilts, moving the poleward edge more poleward than the central cusp region. This is why the slope from the study of Pitout et al. [10] is more than that from our study in this paper.

Choe et al. [5] predicted the dipole tilt angle effect by a simple magnetic dipole model plus magnetopause current. It has also been found that the dayside field-aligned currents (FACs) move poleward and equatorward which should have effect on the variation of the cusp location. Newell et al. [7] supposed that the effect of the dipole tilt angle on the cusp location could be induced by the variation in the dayside current systems with changing dipole tilt angle. One plausible cause of the variation of the currents systems is the variation of the ionospheric conductivity [23,24]. The variation of the dipole tilt angle could control the amount of EUV radiation to reach the ionosphere and thus changing the amount and distribution of conductivity. But Christiansen et al. [25] argued that the seasonal dependence in the global FACs system is generated and maintained by solar windmagnetosphere interaction processes.

Since the intrinsic geomagnetic field is dipole-like, the further away from the Earth, the weak of the magnetic field will be. In other words, the more the altitude is, the weak the magnetic field will be. Therefore, the cusp location is more sensitive if the altitude is higher. Thus, our conclusion that the dependence increases monotonically with the increasing altitude described in Figure 3 is also reasonable from physics. However, the dependence of the cusp location versus altitude still needs to be further studied.

\section{Summary}

Using the data from Cluster, we investigate the dependence of the cusp location on the dipole tilt angle in the mid-altitude cusp region. There are 95 cusp crossings selected to do analysis. The result shows that the center of the cusp region at the altitude of the Cluster orbit is $0.051^{\circ}$ ILAT for every $1^{\circ}$ increase in the dipole tilt angle at the mean altitude of $5.8 \mathrm{R}_{\mathrm{E}}$. It is different from the result obtained by Pitout et al. [10] at nearly the same altitude range because they focus on the poleward boundary of the cusp to study the dependence of the Cusp location. By discussing in physics, the result both from our study and from Pitout et al. [10] is reasonable from physics.

With combination of all dependences so far, from our study with the Cluster data and previous studies by other authors with other different satellite data obtained at different altitudes, we find that the dependence increases monotonically with the increasing altitude, i.e. the higher the altitude is; the larger the dependence (or slope) will be. By fitting, the result shows that the dependence will increase $0.012^{\circ}$ ILAT per degree if the altitude increases $1 \mathrm{R}_{\mathrm{E}}$ in the cusp region. The solar wind interaction with magnetosphere, such as the ram pressure of the solar wind and the IMF orientation (i.e. $B_{y}$ and $B_{x}$ ) could be a direct reason for this result.

Last, it should be mentioned that the cusp is sometimes very dynamical even under northward IMF. Zong et al. $[13,17]$ reported that "multiple" cusps can exist under northward IMF, but it is a very rare phenomenon. In this study, we only take the "single" cusp crossing to do research.

This work was supported by the National Natural Science Foundation of China (41004076, 41274146 and 41074114) and the Specialized Research Fund for State Key Laboratories.

1 Heikkila W J, Winningham J D. Penetration of magnetosheath plasma to low altitudes through the dayside magnetospheric cusps. J Geophys Res, 1971, 76: 883-891

2 Frank L A. Plasma in the earth's polar magnetosphere. J Geophys Res, 1971, 76: 5202-5219

3 Lu J Y, Liu A Q, Kabin K, et al. Three dimensional shape of magnetopause: Global MHD results. J Geophys Res, 2011, 116: A09237

4 Liu Z Q, Lu J Y, Kabin K, et al. Dipole tilt control of the magnetopause for southward IMF from global magnetohydrodynamic simulations. J Geophys Res, 2012, 117: A07207

5 Choe J Y, Beard D B, Sullivan E C. Precise calculation of the magnetosphere surface for a tilted dipole. Planet Space Sci, 1973, 21: 485-498

6 Burch J L. Precipitation of low-energy electrons at high latitudes: Effects of interplanetary magnetic field and dipole tilt angle. J Geophys Res, 1972, 77: 6696-6701

7 Newell P T, Meng C I. Dipole tilt angle effects on the latitude of the cusp and cleft/low-latitude boundary layer. J Geophys Res, 1989, 94: 6949-6953

8 Nĕmeček Z, Merka J, Safrănkovă J. The tilt angle control of the outer cusp position. Geophys Res Lett, 2000, 27: 77-80

9 Zhou X W, Russell C T, Le G, et al. The polar cusp location and its 
dependence on dipole tilt. Geophys Res Lett, 1999, 26: 429-432

10 Pitout F, Escoubet C P, Klecker B, et al. Cluster survey of the midaltitude cusp: 1. Size, location, and dynamics. Ann Geophys, 2006, 24: 3011-3026

11 Newell P T, Sotirelis T, Liou K, et al. Cusp latitude and the optimal solar wind coupling function. J Geophys Res, 2006, 111: A9207

12 Tsyganenko N A, Russell C T. Magnetic signatures of the distant polar cusps: Observations by polar and quantitative modeling. J Geophys Res, 1999, 104: 24939-24955

13 Zong Q G, Fritz T A, Zhang H, et al. Triple cusps observed by Cluster - Temporal or spatial effect? Geophys Res Lett, 2004, 31: L09810

14 Shi Q Q, Pu Z Y, Souceket J, et al. Spatial structures of magnetic depression in the Earth's high-altitude cusp: Cluster multipoint observations. J Geophys Res, 2009, 114: A10202

15 Guo J G, Shi J K, Zhang T L, et al. The correlations of ions density with geomagnetic activity and solar dynamic pressure in cusp region. Chin Sci Bull, 2007, 52: 967-971

16 Zong Q G, Fritz T A, Korth A, et al. Energetic electrons as a field line topology tracer in the high latitude boundary/cusp region: Cluster rapid observations. Surveys Geophys, 2005, 26: 215-240

17 Zong Q G, Fritz T A, Korth A, et al. Multiple cusps during an extended northward IMF period with a significant By component. J Geophys Res, 2008, 113: A01210
18 Balogh A, Carr C M, Acuña M H, et al. The Cluster magnetic field investigation: Overview of in-flight performance and initial results. Ann Geophys, 2001, 19: 1207-1217

19 Rème H, Aoustin C, Bosqued J M, et al. First multi-spacecraft ion measurements in and near the Earth's magnetosphere with the identical Cluster ion spectrometry (CIS) experiment. Ann Geophys, 2001, 19: 1303-1354

20 Johnstone A D, Alsop C, Burge S, et al. Peace: A Plasma electron and current experiment. Space Sci Rev, 1997, 79: 351-398

21 Palmroth M, Laakso H, Pulkkinen T I. Location of high-altitude cusp during steady solar wind conditions. J Geophys Res, 2001, 106: 2110921122

22 Zhao M X, Lu J Y. Nonlinear dispersive scale Alfvén waves in magnetosphere-ionosphere coupling: Physical processes and simulation results. Chin Sci Bull, 2012, 57: 1384-1392

23 Fujii R, Iijima T, Potemra T A, et al. Seasonal dependence of largescale Birkeland currents. Geophys Res Lett, 1981, 8: 1103-1106

24 Ohtani S, Ueno G, Higuchi T, et al. Annual and semi-annual variations of the location and intensity of large-scale field-aligned currents. J Geophys Res, 2005, 110: A01216

25 Christiansen F, Papitashvili V O, Neubert T. Seasonal variations of high-latitude field-aligned currents inferred from Ørsted and Magsat observations. J Geophys Res, 2002, 107: 1029-1035

Open Access This article is distributed under the terms of the Creative Commons Attribution License which permits any use, distribution, and reproduction in any medium, provided the original author(s) and source are credited. 